\title{
Interfacial fracture behaviour between cement paste and epoxy coating - An experimental and phase-field approach
}

\author{
Pratik Gujar ${ }^{10}$, Hirshikesh ${ }^{1}$, Manu Santhanam², Ratna Kumar Annabattula ${ }^{3}$, Pijush Ghosh ${ }^{1 *}$ \\ ${ }^{1}$ Department of Applied Mechanics, Indian Institute of Technology Madras, Chennai, India \\ ${ }^{2}$ Department of Civil Engineering, Indian Institute of Technology Madras, Chennai, India \\ ${ }^{3}$ Department of Mechanical Engineering, Indian Institute of Technology Madras, Chennai, India \\ - Pratik Gujar was invited for submission of this letter as an awardee of the RILEM PhD Grant for the 74th RILEM Annual Week, 2020
}

Received: 19 November 2020 / Accepted: 20 January 2021 / Published online: 11 February 2021

(C) The Author(s) 2021. This article is published with open access and licensed under a Creative Commons Attribution 4.0 International License.

\begin{abstract}
Surface coatings are mainly applied to protect concrete structures against various environmental conditions. However, the interface between the concrete and coating is a weak link, and interfacial delamination is the primary mode of failure. Fracture resistance is the main design criteria for these materials, and therefore, it is necessary to investigate possible causes of interfacial failure for preventive measures. To this end, we have investigated the potential failure mechanisms and measured interfacial strength by performing a pull-off adhesion test on epoxy coated cement paste. From the pull-off adhesion test, adhesive (interface) failure between cement paste and epoxy is predominantly observed. It is observed that the adhesive (interfacial) strength for the sample tested on 50th day is more than the 28 days hydrated sample. This is due to additional curing of cement paste samples after coating epoxy which leads to higher interfacial strength. We also present the phase-field model (PFM) to predict the adhesive failure for this system. The results obtained from the PFM, i.e., load at failure and crack path are validated against experimental results. Finally, the influence of various material parameters such as elastic modulus and fracture energy of the interface on interfacial fracture behaviour is studied.
\end{abstract}

Keywords: Epoxy coated cement paste; Pull-off adhesion test; Interface fracture; Phase-field method

\section{Introduction}

Concrete is the world's most commonly used construction material and is the second most-consumed material after water [1]. Due to the porous nature of concrete, the structures made of concrete deteriorate when exposed to an externally aggressive environment. Surface coatings are used to protect against these different environmental conditions and be the most effective form of protection. The durability of different concrete coatings has been widely studied [2-5]. Almusallam et al. [2] evaluated the durability performance of various types of coatings on concrete exposed to different environmental conditions. The study showed that the epoxy and polyurethane coatings were found to be more effective than other types of organic and inorganic coatings. Berndt et al. [3] investigated the effect of different supplementary cementitious materials, protective coatings, and mortars in improving the resistance to microbiologically influenced corrosion in concrete cooling tower structures at geothermal power plants. Epoxy coatings showed excellent durability under laboratory and field conditions. The performance of concrete-coating systems mainly depends on the interfacial adhesion between them and hence proper adhesion should be ensured for the longevity of composite action. For concrete-coating systems, the interface between concrete and coating is a weak link and hence interfacial delamination is the primary mode of failure observed with this material class. However, there are very limited studies on the interfacial performance of concrete-coating systems. Experimental investigation of performance between various coating materials and cementitious materials needs to be done to investigate the possible causes and the mechanism of failure associated with preventive measures.

As the experimental investigation of interfacial performance is a cumbersome process and also time-consuming, this gives a motivation to apply a numerical method to look into the problem in hand. The cohesive zone method (CZM) [6] within linear elastic fracture mechanics framework (LEFM) has become popular and applied to study the interfacial fracture behaviour between concrete and fiber-reinforced polymer (FRP) [7,8], crack propagation in asphalt concrete [9], metals [10], adhesively bonded joints [11], etc. However, there are very few or almost no studies in the literature to understand the fracture behaviour of a concrete-coating interface system using finite element analysis (numerical analysis). The cohesive zone method requires a predefined location of crack 
to model the failure and an ad-hoc criterion to describe when/where a crack will nucleate. In addition to this, special interface elements need to be inserted into the finite element mesh, which can introduce mesh dependency issue or require a complex algorithm to add interface elements dynamically. Continuum Damage Mechanics (CDM) is a possible alternative to alleviate these issues as it (a) does not require complicated crack tracking algorithms (b) does not require the assumption of a pre-existing crack [12]. However, CDM models involve empirical parameters and excessive mesh dependence of the results due to lack of internal length scale in the formulation. The above limitations are circumvented by the introduction of the phase-field method (PFM) for fracture by the seminal work of Francfort and Marigo [13], and Bourdin et al. [14]. The PFM was originally developed for interface problems and applied to the material solidification process [15]. Within the PFM framework, sharp interfaces are approximated by a scalar field variable that is continuous, which distinguishes between multiple phases within the system.

The scalar field variable is called the phase-field parameter in the fracture context, which is used to describe the completely broken and intact material. Due to its attractive features such as (a) able to deal with complex fracture, crack nucleation, propagation, branching, coalescence, and fragmentation, etc. in a stand-alone variational framework (b) no need to track the crack trajectory even for non-smooth crack surfaces and twisting cracks, the PFM is applied to a wider range of engineering problems, some of which include ductile fracture [16], brittle fracture in functionally graded materials [17], failure in composites and ceramics [18], crack growth in rocks, strength prediction in fiber-reinforced composite and laminated composite, to name a few. Msekh et al. [19] predicted fracture properties of the clay/epoxy nanocomposites system using PFM and studied the influence of interface thickness on tensile strength as well as dissipation energy. In addition to application of PFM to model complex fracture, recent focus is on development of the computationally efficient schemes. Goswami et al. [20] proposed a new physics informed neural network (PINN) algorithm for solving brittle fracture problems. Kristensena and E.Martínez-Pañeda [21] proposed robust and efficient PFM by combining quasi-Newton methods and Monolithic schemes. The monolithic quasi-Newton computation enables to use much larger time increments relative to staggered solution schemes and hence entire simulation time is 10 to 100 times faster than staggered solution schemes. In the cement paste-epoxy composite system, the crack may nucleate at three sites i.e., cement, epoxy or interface between them and subsequently propagates along the interface or within the concrete/epoxy. Predicting these crack nucleation sites are crucial and challenging numerically and hence PFM is used in the present work.

In this study, we have investigated both experimentally and numerically, the possible failure mechanisms associated with epoxy coated cement paste. The epoxy polymer was coated on cement paste and the pull-off adhesion test was used to assess the interfacial strength between them under tensile load. Adhesive (interface) failure between cement paste and epoxy is primarily recorded from the experimental observations. For a better understanding of the failure mechanism, the PFM model by assuming elastic material and brittle fracture is developed and implemented in FEnicS [22], an open-source finite element package. The main objective is to simulate the fracture process of epoxy coated cement paste within the variational framework and analyze the influence of various material parameters such as elastic modulus and fracture energy of the interface on interfacial fracture behaviour.

The paper is organized as follows: Section 2 presents the experimental details. The governing differential equations are briefly presented in Section 3. In Section 4, a simplified finite element model used in this work is presented and results pertaining to both numerical and experimental assessment are discussed in Section 5, followed by major conclusions in the last section.

\section{Experimental work}

We use a pull-off adhesion test as per ASTM D7234-12 [23] to determine the interfacial strength between the cement paste and epoxy coating. The pull-off adhesion test is widely used in the construction field as well as laboratory to measure the bond strength between two materials [3]. The cement paste samples $(60 \mathrm{~mm} \times 60 \mathrm{~mm} \times 15 \mathrm{~mm})$ are cast with a water-cement ratio (w/c ratio) of 0.3 and cured for 28 days. The samples are then coated with epoxy polymer of thickness 300 to 350 microns and allowed to cure for 7 days at the laboratory condition $\left(25 \pm 2^{\circ} \mathrm{C}\right.$ and $70 \% \pm 5$ relative humidity) to ensure the curing of epoxy before performing strength tests. To investigate the effect of curing on interfacial adhesive strength, we prepared another set of samples. The second set of samples are coated on the $28^{\text {th }}$ day and after epoxy curing for 7 days again reintroduced into the curing tank by covering the interface by tape till 50 days from the day of casting before testing. Twelve numbers of representative samples are considered for each day of hydration (i.e., 28 and 50 days) and the samples failed in only interfacial or adhesive mode are considered in the calculation of peak load.

We predominantly observed adhesive (interface) failure between cement paste and epoxy from the pull-off adhesion test, as depicted in Figure 1. This indicates that the cement paste is sufficiently cured, and interfacial strength is the prime concern for design. The average ultimate load at failure was recorded to be $568.63( \pm 103.67) \mathrm{N}$ and $832.52( \pm 131.95) \mathrm{N}$ for 28 and 50-days samples, respectively.

\section{The phase field model formulation}

In this section, we first present the fundamental theory of the phase-field method, where the brittle fracture of material is described by a scalar order parameter, i.e., the phase-field variable, $\phi$. We next present the governing differential equations and the solution methodology. 


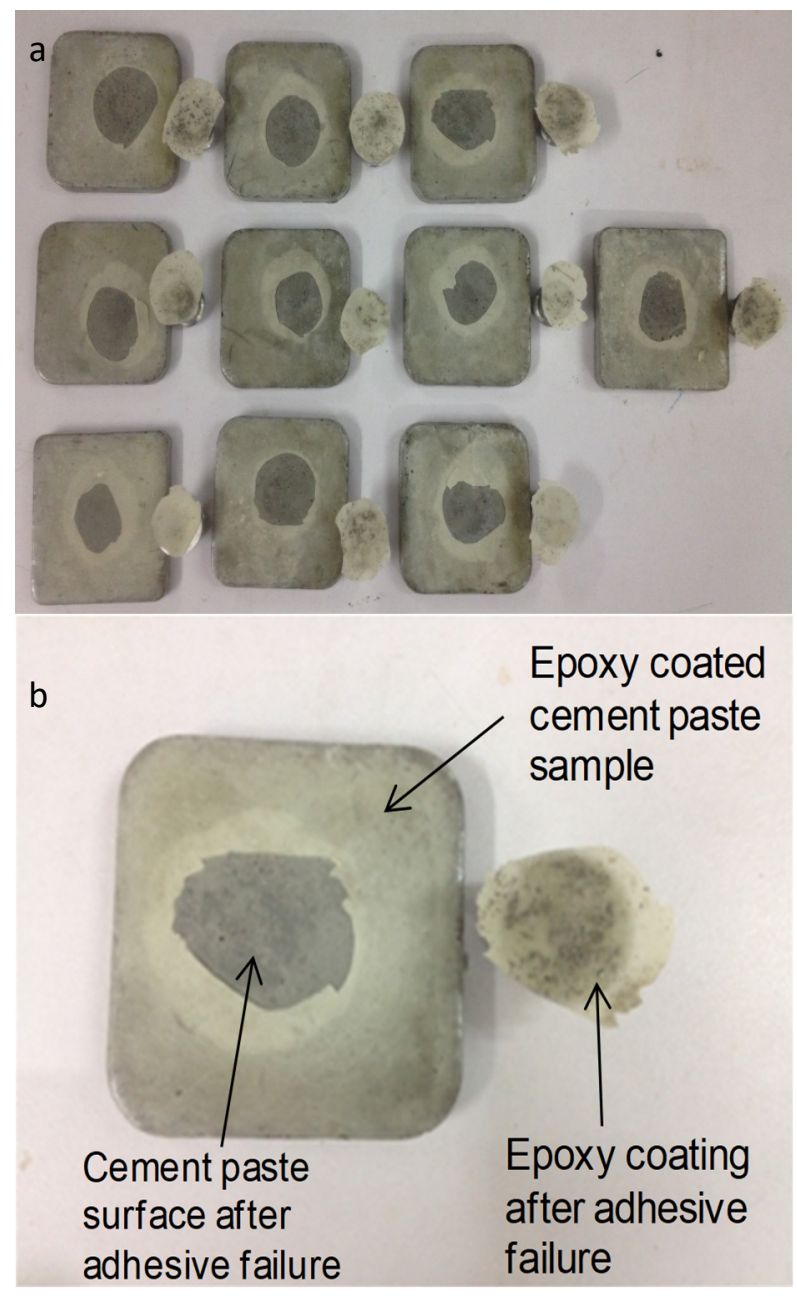

Figure 1. Adhesive (interfacial) failure observed in the pull-off adhesion test experiments for (a) a set of samples considered for 28 days of hydration and $50^{\text {th }}$ day strength tests and (b) individual sample.

\subsection{Phase field formulation for brittle fracture in epoxy coated cement paste}

Within the PFM framework, the strong discontinuity, viz., crack is modeled by a diffused field variable $\phi[0,1]$ (see Figure 2) in which $\phi=0$ represents intact material and $\phi=1$ represents completely damage material, respectively. The crack surface is approximated by defining a regularized crack density functional $\Gamma_{c} \approx \Gamma_{\ell_{o}}\left(\phi, \nabla \phi, \ell_{o}\right)$ [24] which is governed by the choice of length scale, $\ell_{0}$. In this section, only important equations pertaining to the modeling of the strong discontinuity using the PFM are presented. For more detailed discussion, interested readers can refer to the literature $[25,26]$.

Consider a linear elasto-static body with a discontinuity occupying the domain $\Omega \subset \mathbf{R}^{d}$, where $d=2$ is the dimension of the problem. The boundary is considered to admit the decomposition of the boundary $\Gamma$ with outward normal $\mathbf{n}$ into three disjoint sets, i.e., $\Gamma=\Gamma_{D} \cup \Gamma_{N} \cup \Gamma_{C}$ and $\Gamma_{D} \cup \Gamma_{N}=\varnothing$. The closure of the domain is $\bar{\Omega} \equiv \Omega \cup \Gamma$. Let $(\mathbf{u}, \phi): \Omega \rightarrow \mathbf{R}^{d}$ be the displacement field and the scalar damage variable at a point $\mathbf{x}$ when subjected to external tractions $\bar{t}$ on $\Gamma_{N}$ and imposed essential boundary conditions $(\widehat{\mathbf{u}}, \hat{\phi})$ on $\Gamma_{D}$ (see Figure 2 ).

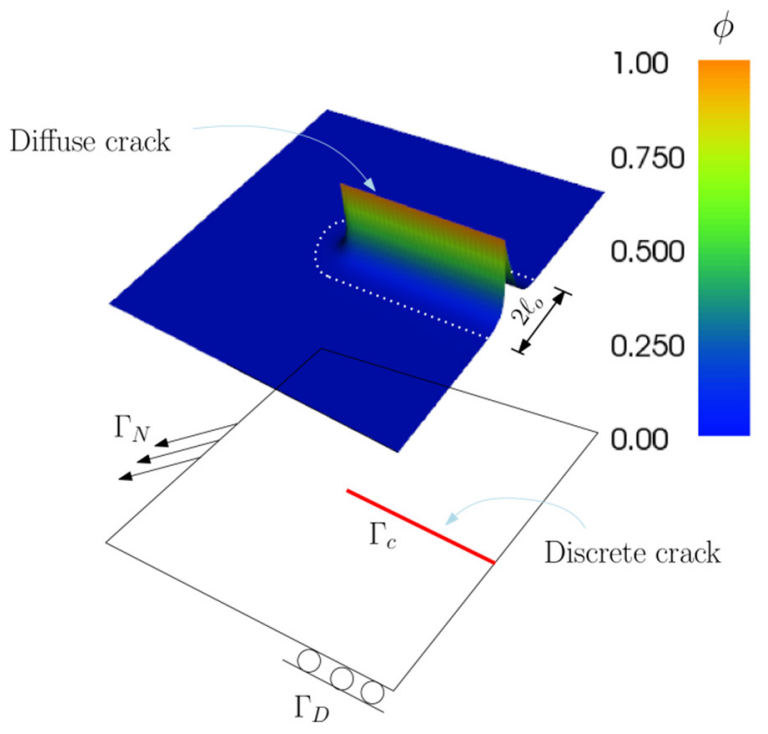

Figure 2. Schematic representation of the crack using discrete and diffuse approach. Within PFM framework scalar field variable, $\phi$ is used to represent the crack. Note that width of the diffuse crack is controlled by the characteristic length scale, $\ell_{o}$.

The boundary value problem in the absence of body force is then: find $(\mathbf{u}, \phi): \Omega \rightarrow \mathbf{R}^{d}$ such that:

$$
\begin{gathered}
\nabla \cdot \boldsymbol{\sigma}=\mathbf{0} \text { in } \Omega, \\
-G_{c} \ell_{o} \nabla^{2} \phi+\left[\frac{G_{c}}{\ell_{o}}+2 H\right] \phi=2 H \text { in } \Omega,
\end{gathered}
$$

with the following boundary conditions:

$$
\begin{array}{r}
\nabla \cdot \boldsymbol{\sigma}=\overline{\mathbf{t}} \text { on } \Gamma_{N}, \\
\mathbf{u}=\overline{\mathbf{u}} \text { on } \Gamma_{D}, \\
\nabla \phi \cdot \mathbf{n}=0 \text { on } \Gamma_{N},
\end{array}
$$

where $\boldsymbol{\sigma}=\left[(1-\phi)^{2}+k\right] \frac{\partial \psi(\varepsilon)}{\partial \varepsilon}$ is the Cauchy stress tensor and $\psi(\varepsilon)=\frac{1}{2} \lambda(\operatorname{tr}(\varepsilon))^{2}+\mu \operatorname{tr}\left(\varepsilon^{2}\right)$ is the elastic energy density with $\mu$ and $\lambda$ the Lamé constants, $\varepsilon=$ $\frac{1}{2}\left[\nabla u+\nabla u^{T}\right]$ is the small strain tensor, $k$ is introduced for numerical stability, and the history variable $H$ is defined as:

$$
H=\left\{\begin{array}{c}
\psi(\boldsymbol{\varepsilon}), \quad \psi(\boldsymbol{\varepsilon})<H_{n}, \\
H_{n}, \text { otherwise }
\end{array}\right.
$$

where $H_{n}$ is the strain energy computed at load step $n$. The above model fails to distinguish between the crack growth due to local tensile and compressive stress state. In order to prevent the evolution of phase field variable in the localized compressive region, Amor et al. [27] proposed additive decomposition of elastic energy based on volumetric and deviatoric part and Miehe et al. [28] proposed spectral decomposition of the elastic energy density. These approaches lead to a non-linear system of equations for the elasticity as a result of the decomposition of the strain tensor. This could be computationally expensive. In order to alleviate this, Ambati et al. [25] introduced a hybrid approach in which the linear momentum equation (Equation 1 ) is retained the 
same as the isotropic model and the evolution of the phasefield variable is controlled by the tensile elastic energy $\psi^{\ddagger}$ alone. This condition ensures that the phase field variable does not evolve in the compressive regions and the linear momentum equation (see Equation 1) is linear which reduces the computational complexity. The modified phase-field equation is given by:

$$
-G_{c} \ell_{o} \nabla^{2} \phi+\left[\frac{G_{c}}{\ell_{o}}+2 H^{+}\right] \phi=2 H^{+ \text {in } \Omega}
$$

where $H^{+}:=\max _{\tau \in[0, t]} \psi^{+}(\boldsymbol{\varepsilon}(\mathbf{x}, \tau)) \cdot$

Equation (4) is supplemented with the following constraint to prevent crack face inter penetration:

$$
\forall \mathbf{x}: \psi^{+}<\psi^{-} \Rightarrow \phi:=0
$$

and

$$
\psi^{ \pm}(\boldsymbol{\varepsilon})=\frac{1}{2} \lambda\langle\operatorname{tr}(\boldsymbol{\varepsilon})\rangle_{ \pm}^{2}+\mu \operatorname{tr}\left(\boldsymbol{\varepsilon}_{ \pm}^{2}\right)
$$

with

$$
\boldsymbol{\varepsilon}_{ \pm}=\sum_{I=1}^{3}\left\langle\varepsilon_{I}\right\rangle_{ \pm} \mathbf{n}_{I} \otimes \mathbf{n}_{I} \quad \text { and } \varepsilon=\sum_{I=1}^{3} \boldsymbol{\varepsilon}_{I} \mathbf{n}_{I} \otimes \mathbf{n}_{I}
$$

where $\varepsilon_{I}$ and $\mathbf{n}_{I}$ are the principal strains and principal strain directions, respectively.In this work, Equations (1) and (7) are discretized using finite element method and solved using a staggered solver, wherein, we solve for the phase-field order parameter $(\phi)$ using the displacement field $(\mathbf{u})$. Then, the updated phase-field order parameter is used to solve for the displacement field. Interested readers can refer to Hirshikesh et al. [29] for more details for implementation and solution algorithm.

\section{Model}

To numerically investigate the fracture process of epoxy coated cement paste, a two-dimensional finite element model for the pull-off adhesion test is developed. To take the advantage of symmetry, only half of the model is considered in the present simulations. The domain and the boundary conditions for the boundary value problem are shown in
Figure 3. In order to simulate uniaxial tension, the bottom edge is constrained in the $y$-direction and the right edge is constrained in the $x$-direction. An incremental uniform displacement is applied on the top surface after $2.5 \mathrm{~mm}$ from the left edge to avoid boundary effects (see Figure 3 ). The interface between cement paste and epoxy is considered as a separate layer with the thickness equal to one-tenth of the thickness of the epoxy coating, $t_{E}$ (see Figure 3). One of the aims of the present study is to understand the role of the interface on fracture strength of the epoxy-cement system, and hence the interface is explicitly modeled in the current analysis. Also, to make the model computationally efficient, only some part of the cement paste i.e., 5 times the thickness of the epoxy coating is considered for modeling.This assumption is valid as fracture takes place near the interface. For this work, the surface of cement paste is considered to be ideally flat and a perfect bond between cement paste and the epoxy coating is assumed.

The details of the material properties used for the simulation are presented in Table 1. The elastic modulus $E$ for 28 and 50 days cement paste is calculated from their characteristic compressive strengths by using empirical relation $E=$ $5000 \sqrt{f_{c k}}$ where $f_{c k}$ is the characteristic compressive strength [30]. The fracture energy and Poisson's ratio for cement paste are taken as a reference from the literature [31,32].The elastic modulus and fracture energy for the epoxy polymer are also taken as a reference from literature [33]. To simulate adhesive failure, the elastic modulus of the interface is varied by considering three cases: (a) elastic modulus of interface $\left(E_{1}\right)$ equal to the elastic modulus of epoxy $\left(E_{\mathrm{E}}\right)(\mathrm{b})$ elastic modulus of interface $\left(E_{1}\right)$ equal to the elastic modulus of cement paste $\left(E_{C}\right)$, and (c) elastic modulus of interface equal to the average of elastic modulus of epoxy and cement paste. Out of three cases, case (a) shows interface (adhesive) failure while the other two failed to simulate adhesive failure. The influence of elastic modulus of the interface on interfacial fracture is discussed in detail in section 5.2.

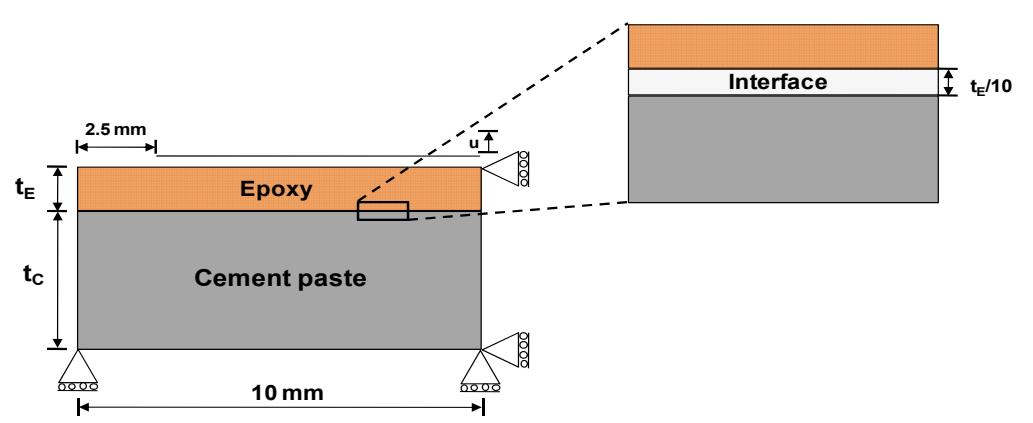

Figure 3. Schematic of the FE model for the pull-off adhesion test. Where $t_{E}=0.350 \mathrm{~mm}, t_{c}=1.75 \mathrm{~mm}$. 


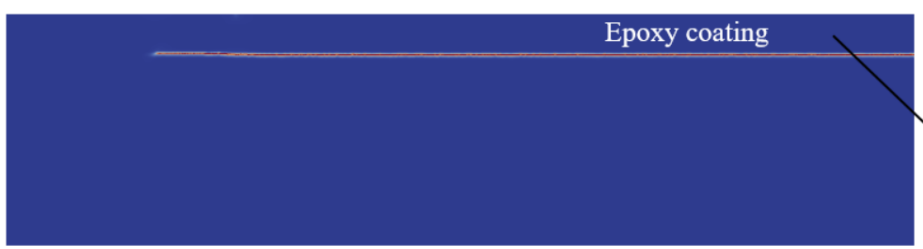

(a)

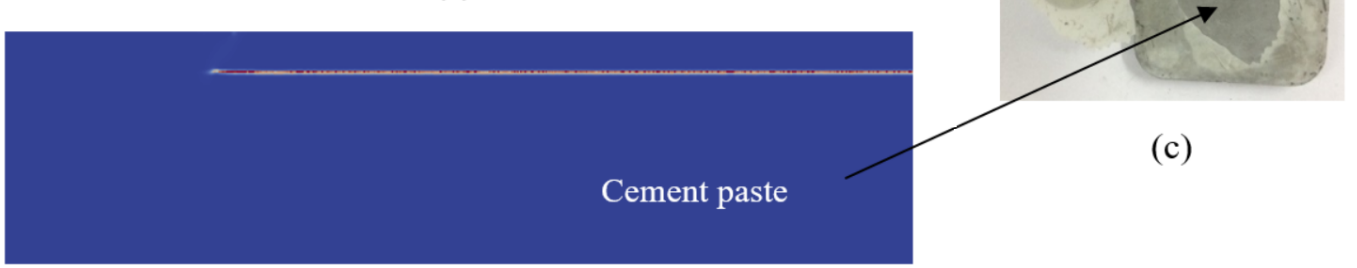

(b)

Figure 4. Crack trajectory comparison for (a) 28-days, (b) 50-days hydrated cement paste samples using PFM and (c) experiment. The phase-field model predicts adhesive failure which was predominantly observed in the pull-off adhesion test experiments.

The fracture energy of the interface is calculated from the load-displacement response obtained from the slant shear test experiments by considering brittle fracture. The area under the load-displacement curve is the measure of fracture energy. The values obtained were used as a reference for the pull-off adhesion test. The fracture energy in Mode II is assumed to be nearly 10 to 15 times the fracture energy in Mode I [34]. Therefore, in this work, to simulate adhesive failure, the fracture energy of the interface is considered to be 10 times lesser than the fracture energy obtained from the slant shear test.

Table 1. Material properties of cement paste, interface, and epoxy polymer.

\begin{tabular}{|l|l|l|l|}
\hline Material & $\begin{array}{l}\text { Elastic Modulus } \\
\text { (MPa) }\end{array}$ & $\begin{array}{l}\text { Poisson's } \\
\text { Ratio }\end{array}$ & $\begin{array}{l}\text { Fracture Energy } \\
\left(\mathrm{J} / \mathrm{m}^{2}\right)\end{array}$ \\
\hline $\begin{array}{l}\text { Cement } \\
\text { paste } \\
\text { (28days) }\end{array}$ & 35000 & $0.24[32]$ & $25[31]$ \\
\hline $\begin{array}{l}\text { Cement } \\
\text { paste } \\
\text { (50 }\end{array}$ & 40000 & $0.24[32]$ & $30[31]$ \\
\hline $\begin{array}{l}\text { Interface } \\
\text { day }\end{array}$ & 3500 & 0.25 & $\begin{array}{l}40.5 \text { (for 28 days) } \\
57.6 \text { (for 50 days) }\end{array}$ \\
\hline Epoxy & $3500[33]$ & 0.30 & $280[33]$ \\
\hline
\end{tabular}

\section{Results and discussion}

In this section, we present the results for the study of the epoxy coated cement paste system in terms of interfacial fracture behaviour. Mainly, the fracture patterns i.e., crack nucleation at the interface and subsequent propagation and adhesive strength are presented. Section 5.1 presents the feasibility of the PFM to simulate fracture behaviour of the epoxy coated cement paste system and then understanding is gained in terms of crack trajectory and load-carrying capacity of the system by varying the interface properties in Section 5.2. The domain is discretized (generated unstructured mesh using Gmsh and converted to ".xml" format to be used in FEniCS) with linear triangular elements with at least 6 elements through the interface thickness. The characteristic length $\ell_{o}$ for all the problems is chosen as $\ell_{o}=2 h$ unless stated otherwise, where $h$ is the size of the smallest element in the domain. The existing constraint on discretization results in a smaller length scale than the computed length scale from material properties, as $\ell_{0}=$ $\frac{27 \mathrm{EG}_{\mathrm{c}}}{526 \sigma_{\mathrm{c}}^{2}}$ [35] and shows mesh independent load-displacement response.

\subsection{Model validation against experimental results}

First, we validated the PFM model against the experimental results of the pull-off adhesion test. Due to the application of the uniform displacement at the top surface, the crack nucleates at the interface and subsequently propagates along the interface (see Figure 4). The red line shows the complete failure of the material $(\phi=1)$ and the blue region $(\phi=0)$ shows the intact state of the material. Note that there is a smooth transition from blue to red color, which describes the diffused crack as mentioned in Section 3.1. The crack trajectory is in close agreement with the experimental observation as shown in Figure 4.

The load-carrying capacity of the system is also analyzed in terms of the reaction force. From the pull-off adhesion test experiments, ultimate strength at failure is recorded and the ultimate load was calculated by multiplying ultimate strength by the area of the dolly (diameter: $20 \mathrm{~mm}$ ). Figure 5 compares the load-displacement response for 28 - and 50 -days hydrated cement paste for different characteristic lengths. The characteristic length scale $\ell_{0}=0.0023$ is sufficient to predict experimental results. Though the crack trajectory is similar for 28-and 50-days samples, the average load-carrying capacity for the 50 -days sample is more than the 28-days. This gain in the load-carrying capacity is due to additional curing of cement paste samples after coating of epoxy which leads to higher interfacial strength. Table 2 compares the peak load obtained from PFM and pull-off adhesion test experiments. 
The differences in the results of peak load obtained from PFM and pull-off adhesion test experiments may be attributed to the assumption of elastic materials and brittle fracture for both epoxy and cement paste. Another reason may be the assumption of an ideal flat surface of the cement paste and the perfect bond between cement paste and epoxy coating. However, in reality, the cement paste surface is not flat because of the inherent surface roughness.

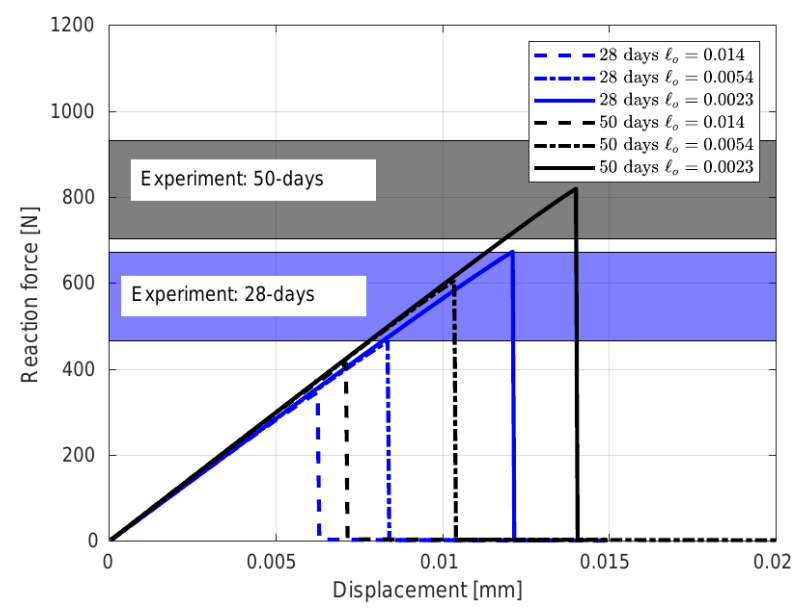

Figure 5. Load displacement response for 28-days and 50-days hydrated cement paste for different length scale parameters.

Once the model validation against experimental results is completed, conducting a parametric study is essential to investigate the influence of each input parameter on the physical behaviour of the system. Therefore, the influence of material parameters such as elastic modulus and fracture energy of the interface on interfacial performance between cement paste and epoxy is studied.

Table 2. Comparison of peak load from pull-off test experiments and PFM.

\begin{tabular}{|c|c|c|c|}
\hline & \multicolumn{2}{|c|}{$\begin{array}{l}\text { Peak load in } \mathrm{N} \\
\text { (Standard deviation in } \\
\text { parentheses) }\end{array}$} \\
\hline & & 28 Days & 50 Days \\
\hline \multicolumn{2}{|c|}{ Experiment } & $\begin{array}{l}568.63 \\
( \pm 103.67)\end{array}$ & $\begin{array}{l}832.52 \\
( \pm 131.95)\end{array}$ \\
\hline \multirow{3}{*}{ PFM } & $\begin{array}{l}\text { Upper bound } \\
\text { of fracture energy }\end{array}$ & 600.21 & 819.82 \\
\hline & $\begin{array}{l}\text { Middle bound of } \\
\text { fracture energy }\end{array}$ & 498.09 & 686.83 \\
\hline & $\begin{array}{l}\text { Lower bound } \\
\text { of fracture energy }\end{array}$ & 376.02 & 531.12 \\
\hline
\end{tabular}

\subsection{Influence of elastic modulus and fracture energy of the interface on interfacial fracture behaviour}

Now, understanding of the adhesive failure and adhesive strength is gained under the influence of different material properties of the interface. The study is helpful for the better design of the interface with higher fracture resistance. To this end, we have investigated the role of elastic modulus and fracture energy of the interface on the crack path and loaddisplacement response of the cement paste-epoxy coating system. The properties of the interface are varied by considering three different cases:

- Case I: Elastic modulus of interface $\left(E_{I}\right)$ equal to the elastic modulus of epoxy $\left(E_{E}\right)$ i.e., $E_{I}=E_{E}$,

- Case II: Elastic modulus of interface $\left(E_{I}\right)$ equal to the elastic modulus of cement paste $E_{C}$ i.e., $E_{I}=E_{C}$, and

- Case III: Elastic modulus of interface $\left(E_{I}\right)$ equal to the average of elastic modulus of epoxy and cement paste i.e., $E_{I}=\frac{E_{E}+E_{C}}{2}$.

Adhesive (interface) failure between cement paste and epoxy was observed for case I (see Figure 4). This can be attributed to the (a) lower fracture energy of the interface (refer Table 1) as compared to the epoxy, (b) lower elastic modulus of the interface as compared to cement paste (though the fracture energy is nearly the same). On the other hand, case II and III showed the cohesive failure of the epoxy polymer. The cohesive failure of epoxy polymer is mainly because of the lower value of the elastic modulus of the epoxy as compared to cement paste although the fracture energy is very high in comparison with cement paste and interface. This condition forces the epoxy polymer to fail first. Hence, it can be concluded that the type of failure is governed by both fracture energy as well as elastic modulus of the material.

Now, to investigate the effect of fracture energy on the loaddisplacement response, fracture energy of the interface is varied from $23.3 \mathrm{~J} / \mathrm{m}^{2}$ to $57.6 \mathrm{~J} / \mathrm{m}^{2}$, which is the lower and upper bound of the fracture energy obtained from the experimental load-displacement response. For the range of fracture energy values considered, only the adhesive failure was primarily observed. But the lower bound fracture energy value under-predicts the value of the peak load at failure while the peak load obtained from upper bound fracture energy nearly matches with the experimental peak load values. The comparison of peak load obtained from PFM and pull-off adhesion test experiments with upper bound and lower bound of the fracture energy are shown in Table 2 . Beyond the lower and upper bound of the fracture energy considered, other types of failures, such as the cohesive failure of epoxy polymer may become dominant. Hence, critical fracture energy is a key factor for the interfacial fracture behaviour between cement paste and the epoxy coating. From the results of numerical analysis, it can be concluded that the elastic modulus and fracture energy governs the interfacial fracture behaviour between cement paste and epoxy coating. Also, the types of failures (adhesive or cohesive failure of epoxy polymer) mainly depend on the elastic modulus and fracture energy of the material.

\section{Conclusions}

The phase-field method is used to investigate the interfacial fracture behaviour between cement paste and epoxy coating. First, the model is developed and validated against the experimental results of the pull-off adhesion test. The present PFM formulation is proven to be capable of predicting the crack path i.e., crack initiation and subsequent propagation, and also the load-displacement response. The differences in 
the results of peak load obtained from PFM and pull-off adhesion test experiments may be attributed to the assumption of (a) elastic materials and brittle fracture for both cement paste and epoxy (b) ideal flat surface of the cement paste and the perfect bond between cement paste and epoxy coating. Finally, the elastic modulus and fracture energy of the interface are varied to investigate their effect on the crack path and the load-carrying capacity. The results obtained provide fundamental and quantitative insight into the role of the elastic modulus and fracture energy of the interface on the fracture path and load-carrying response of the cement paste-epoxy coating system. The results obtained can be used as a guideline to design high fracture resistance epoxy coated cement paste. The presented PFM approach can be applied for the prediction of interfacial fracture behaviour between two materials such as a cement pasteepoxy coating system. The results presented in this work may be further improved by incorporating non-smooth interface and viscoelastic response of the polymer material. Furthermore, the development of an empirical relation describing the dependence of fracture properties on the curing time has the potential to serve as a useful design tool in future.

\section{CRediT authorship statement}

Pratik Gujar: Conceptualization, Methodology, Data curation, Investigation, Validation, Writing - original draft.

Hirshikesh: Conceptualization, Methodology, Software, Investigation, Writing - original draft.

Manu Santhanam: Conceptualization, Resources, Supervision.

Ratna Kumar Annabattula: Conceptualization, Resources, Editing the draft, Supervision.

Pijush Ghosh: Conceptualization, Resources, Editing the draft, Supervision.

\section{Declaration of competing interest}

The authors declare that they have no known competing financial interests or personal relationships that could have appeared to influence the work reported in this paper.

\section{References}

[1] CR. Gagg, Cement and concrete as an engineering material: An historic appraisal and case study analysis. Eng Fail Anal (2014) 40:114140. https://doi.org/10.1016/j.engfailanal.2014.02.004

[2] AA. Almusallam, FM. Khan, SU. Dulaijan, OSB. Al-Amoudi, Effectiveness of surface coatings in improving concrete durability. Cem Concr Compos (2003) 25:473-481. https://doi.org/10.1016/S0958-9465(02)00087-2

[3] ML. Berndt, Evaluation of coatings, mortars and mix design for protection of concrete against sulphur oxidising bacteria. Constr Build Mater (2011) 25:3893-3902. https://doi.org/10.1016/j.conbuildmat.2011.04.014

[4] AR. Suleiman, AM. Soliman, ML. Nehdi, Effect of surface treatment on durability of concrete exposed to physical sulfate attack. Constr Build Mater (2014) 73:674-681. https://doi.org/10.1016/j.conbuildmat.2014.10.006

[5] A. Arabzadeh, H. Ceylan, S. Kim, K. Gopalakrishnan, A. Sassani, S. Sundararajan, PC. Taylor, Superhydrophobic coatings on portland cement concrete surfaces. Constr Build Mater (2017) 141:393-401. https://doi.org/10.1016/j.conbuildmat.2017.03.012
[6] CV. Verhoosel, MA. Scott, R. de Borst, TJR. Hughes, An isogeometric analysis to cohesive zone modelling. Int J Numer Methods Eng (2011) 87:336-360. https://doi.org/10.1002/nme.3061

[7] YT. Obaidat, S. Heyden, O. Dahlblom, The effect of CFRP and CFRP/concrete interface models when modelling retrofitted RC beams with FEM. Compos Struct (2010) 92:1391-1398. https://doi.org/10.1016/j.compstruct.2009.11.008

[8] K Park, K. Ha, H Choi, C. Lee, Prediction of interfacial fracture between concrete and fiber reinforced polymer (FRP) by using cohesive zone modelling. Cem Concr Compos (2015) 63:122-131. https://doi.org/10.1016/i.cemconcomp.2015.07.008

[9] SH. Song, GH. Paulino, WG. Buttlar, Simulation of crack propagation in asphalt concrete using an intrinsic cohesive zone model. J Eng Mech (2006) 132:1215-1223. https://doi.org/10.1061/(ASCE)0733-9399(2006)132:11(1215)

[10] F. Javidrad, M. Mashayekhy, A cohesive zone model for crack growth simulation in AISI 304 steel. J Solid Mech (2014) 6(4):378-388.

[11] RJB. Rocha, RDSG. Campilho, Evaluation of different modelling conditions in the cohesive zone analysis of single-lap bonded joints. J Adhes (2018) 94:562-582. https://doi.org/10.1080/00218464.2017.1307107

[12] RHJ. Peerlings, R de Borst, WAM. Brekelmans, MGD. Geers, Gradient enhanced damage modelling of concrete fracture. Mech CohesiveFrictional Mater (1998) 3:323-342. https://doi.org/10.1002/(SICl)1099-1484(1998100)3:4<323::AIDCFM51>3.0.CO;2-Z

[13] B. Bourdin, GA. Francfort, J. Marigo, Numerical experiments in revisited brittle fracture. J Mech Phy Solids (2000) 48:797-826. https://doi.org/10.1016/S0022-5096(99)00028-9

[14] B. Bourdin, GA. Francfort, J. Marigo, The Variational Approach to Fracture. Springer Netherlands (2008). https://doi.org/10.1007/978-1-4020-6395-4

[15] JW. Cahn, JE. Hilliard, Free energy of a nonuniform system. I. interfacial free energy. J Chem Phys (1958) 28(2):258-267. https://doi.org/10.1063/1.1744102

[16] M. Ambati, R. Kruse, L. De Lorenzis, A phase-field model for ductile fracture at finite strains and its experimental verification. Comput Mech (2016) 57:149-167. https://doi.org/10.1007/s00466-015-1225-3

[17] Hirshikesh, S. Natarajan, RK. Annabattula, E. Martinez-Paneda, Phase field modelling of crack propagation in functionally graded materials. Compos Part B Eng (2019) 169:239-248. https://doi.org/10.1016/j.compositesb.2019.04.003

[18] Hirshikesh, S. Natarajan, RK. Annabattula, Modeling crack propagation in variable stiffness composite laminates using the phase field method. Compos Struct (2019)209:424-433. https://doi.org/10.1016/j.compstruct.2018.10.083

[19] MA. Msekh, NH. Cuong, G. Zi, P. Areias, X. Zhuang, T.Rabczuk, Fracture properties prediction of clay/epoxy nanocomposites with interphase zones using a phase field model. Eng Fract Mech (2018) 188: 287-299. https://doi.org/10.1016/j.engfracmech.2017.08.002

[20] S. Goswami, C.Anitescu, S.Chakraborty, T.Rabczuk, Transfer learning enhanced physics informed neural network for phase-field modeling of fracture. Theor Appl Fract (2020) 106:102447. https://doi.org/10.1016/j.tafmec.2019.102447

[21] PK.Kristensen, E. Martínez-Pañeda, Phase field fracture modelling using quasi-Newton methods and a new adaptive step scheme. Theor Appl Fract (2020) 107:102446. https://doi.org/10.1016/i.tafmec.2019.102446

[22] MS. Alnæs, J. Blechta, J. Hake, A. Johansson, B. Kehlet, A. Logg, C. Richardson, J. Ring, ME. Rognes, GN. Wells, The FEniCS Project Version 1.5, Arch. Numer. Softw. 3 (2015).

[23] ASTM D7234 - 19 Standard Test Method for Pull-Off Adhesion Strength of Coatings on Concrete Using Portable Pull-Off Adhesion Testers.

[24] B. Bourdin, GA. Francfort, JJ. Marigo, Numerical experiments in revisited brittle fracture. J Mech Phys Solids (2000) 48:797-826. https://doi.org/10.1016/S0022-5096(99)00028-9

[25] M. Ambati, T. Gerasimov, LD.Lorenzis, A review on phase-field models of brittle fracture and a new fast hybrid formulation. Comput Mech (2015) 55:383-405. https://doi.org/10.1007/s00466-014-1109-y

[26] JY. Wu, VP. Nguyen, CT. Nguyen, D. Sutula, S. Sinaie, S. Bordas, Phasefield modeling of fracture. Adv Appl Mech (2020) 53:1-183. https://doi.org/10.1016/bs.aams.2019.08.001

[27] H. Amor, JJ. Marigo, C. Maurini, Regularized formulation of the variational brittle fracture with unilateral contact: Numerical 
experiments. J Mech Phys Solids (2009) 57: 1209-1229. https://doi.org/10.1016/j.jmps.2009.04.011

[28] C. Miehe, M. Hofacker, F. Welschinger, A phase field model for rateindependent crack propagation: Robust algorithmic implementation based on operator splits. Comput Methods Appl Mech Eng (2010) 199:2765-2778. https://doi.org/10.1016/j.cma.2010.04.011

[29] Hirshikesh, S. Natarajan, RK. Annabattula, A FEniCS implementation of the phase field method for quasi-static brittle fracture. Front Struct Civ Eng (2019)13:380-396.

https://doi.org/10.1007/s11709-018-0471-9

[30] IS 456, Plain and Reinforced Concrete-Code of Practice, Bur. Indian Stand. (2000) 16.

[31] SL. Xu, Y. Zhu, Experiment studies on fracture energy of cement paste and mortar. Key Eng Mater (2007) 348:169-172.

https://doi.org/10.4028/www.scientific.net/KEM.348-349.169

[32] I. Maruyama, G. Igarashi, Cement reaction and resultant physical properties of cement paste. J Adv Concr Technol (2014) 12:200-213. https://doi.org/10.3151/jact.12.200

[33] P. Dittanet, RA. Pearson, Effect of silica nanoparticle size on toughening mechanisms of filled epoxy. Polymer (2012) 53:18901905. https://doi.org/10.1016/i.polymer.2012.02.052

[34] P Neto, J Alfaiate, JR Almeida, EB Pires, The influence of mode II fracture on concrete strengthened with CFRP. Comput Struct (2004) 82:1495-502. https://doi.org/10.1016/j.compstruc.2004.03.045

[35] E. Martínez-Pañeda, A. Golahmar, CF.Niordson, A phase field formulation for hydrogenassisted cracking. Comput Methods Appl Mech Eng (2018) 342:742-761.

https://doi.org/10.1016/j.cma.2018.07.021 